\title{
Rigorous Analytical Model for Metasurface Microscopic Design with Interlayer Coupling
}

\author{
Shahar Levy, Yaniv Kerzhner and Ariel Epstein*, \\ Andrew and Erna Viterbi Faculty of Electrical Engineering, Technion-Israel Institute of Technology, Haifa 3200003, Israel \\ Email: *epsteina@ee.technion.ac.il
}

\begin{abstract}
We present a semianalytical method for designing meta-atoms in multilayered metasurfaces (MSs), relying on a rigorous model developed for multielement metagratings. Notably, this model properly accounts for near-field coupling effects, allowing reliable design even for extremely small interlayer spacings, verified via commercial solvers. This technique forms an appealing alternative to the common full-wave optimization employed for MS microscopic design to date.
\end{abstract}

\section{INTRODUCTION}

In recent years, numerous reports have demonstrated the ability of Huygens' and bianisotropic metasurfaces (MS) to perform complex wavefront manipulation at microwave frequencies [1], [2]. These planar structures are composed of subwavelength polarizable elements (meta-atoms) featuring electric, magnetic, and (possibly) magnetoelectric responses. Synthesis of such MSs usually includes a macroscopic design stage, where the necessary homogenized MS constituent distribution is derived from the desired field transformation; and a microscopic design stage, where this abstract distribution is discretized, and physical structures for the metaatoms implementing the required local response are devised [3]. The latter typically relies on time-consuming full-wave optimization; although alternative semianalytical transmissionline-model approaches have been proposed for the common cascaded impedance sheet formation [4], these techniques neglect interlayer near-field coupling effects, thus require a final optimization step in full-wave solvers [5].

In this paper, we present a rigorous reliable microscopic design method to synthesize a multilayer MS, composed of $N$ cascaded capacitively-loaded wires (Fig. 1). The method relies on the analytical model presented in [6] and adjusted for metagratings [7], allowing characterization of the metaatom response in a way that properly accounts for the nearfield phenomena. We utilize this method to semianalytically compose a look-up table (LUT) for 5-layer Huygens' metaatoms, used to design a Huygens' MS (HMS) for anomalous refraction. Simulations in a commercial solver verify that, even for extremely close interlayer separation, highly-efficient refraction is obtained, without any full-wave optimization.

\section{THEORY}

We consider multilayered meta-atoms composed of $N$ cascaded wires, loaded with printed capacitors of width $W_{n}$, positioned at the planes $z=n h(0 \leq n<N)$, and designated to be excited with transverse electric fields $\left(E_{z}=E_{y}=H_{x}=0\right)$.

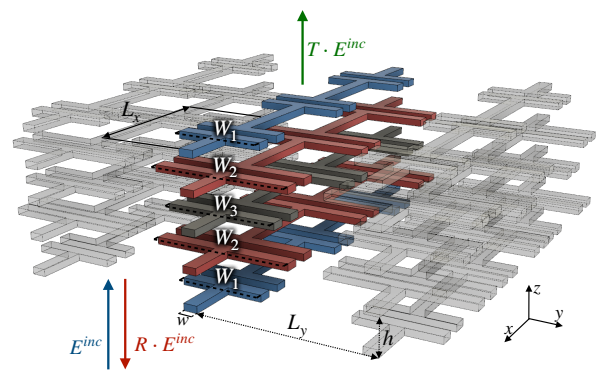

Fig. 1. Multilayered meta-atom characterization configuration $(N=5)$.

To associate a given physical structure with effective polarizabilities (thus compiling the aforementioned LUT), the $L_{x} \times L_{y}$ meta-atoms are repeated periodically along the $x$ and $y$ axes $\left(L_{x}, L_{y} \ll \lambda\right)$, and their response to a normally incident plane wave $E_{x}^{\text {inc }}(\vec{r})=E_{\text {in }} e^{-j k z}$ is evaluated (Fig. 1] [3].

When excited by $E_{x}^{\text {inc }}$, currents will be induced on the wires at the $n$th layer; as $L_{x} \ll \lambda$, these can be effectively treated as electric line sources carrying current $I_{n}$ parallel to the $x$ axis, uniformly loaded by an impedance density of $\tilde{Z}_{n}$ (associated with the printed capacitor of width $\left.W_{n}\right)[6]-[8]$. Consequently, the fields produced by the excited wires at $z=n h$ read

$$
E_{x, n}^{\mathrm{ls}}(\vec{r})=-\frac{k \eta}{4} I_{n} \sum_{p=-\infty}^{\infty} H_{0}^{(2)}\left[k \sqrt{\left(y-p L_{y}\right)^{2}+(z-n h)^{2}}\right]
$$

where the induced currents $I_{n}$ need to be computed to assess the scattered fields. This can be achieved by invoking Ohm's law on the wires; using the Poisson formula, one gets [6], [7]

$$
\begin{aligned}
\tilde{Z}_{n} I_{n} & =E_{\text {in }} e^{-j k n h}+j \frac{k \eta}{2 \pi} I_{n} \log \left(\frac{\pi w}{2 L_{y}}\right) \\
& -\frac{\eta}{2 L_{y}}\left(\sum_{l=0}^{N-1} I_{l} e^{-j k|n-l| h}\right) \\
& -j k \eta \sum_{m=1}^{\infty}\left(\frac{\sum_{l=0}^{N-1} I_{l} e^{-\alpha_{m}|n-l| h}}{L_{y} \alpha_{m}}-\frac{I_{n}}{2 \pi m}\right)
\end{aligned}
$$

where $w$ is the wire width and $\alpha_{m}=\sqrt{\left(2 \pi m / L_{y}\right)^{2}-k^{2}}$.

For given meta-atom geometry, i.e. given load impedances $\tilde{Z}_{n}$, (2) forms a set of linear equations, from which the currents $I_{n}$ can be resolved. Using these, the total scattered fields can be evaluated by summing the contributions (1) of the $N$ cascaded layers and the incident field. Finally, with the Poisson 
formula, the total fields can be expressed as a sum of FloquetBloch (FB) modes, from which the homogenized (zeroth-order mode) transmission and reflection coefficients associated with the given meta-atom can be readily retrieved as [3], [6]

$$
T=1-\frac{\eta}{2 L_{y}} \sum_{n=0}^{N-1 \frac{I_{n}}{E_{\text {in }}}} e^{j k n h}, \quad R=\frac{\eta}{2 L_{y}} \sum_{n=0}^{N-1 \frac{I_{n}}{E_{\text {in }}}} e^{-j k n h}
$$

\section{RESULTS AND DISCUSSION}

To verify and demonstrate the prescribed formalism, we utilize it to design a HMS for anomalous refraction, redirecting a normally incident plane wave towards $\theta_{\text {out }}=56^{\circ}$. The MS is composed of a symmetric cascade of $N=5$ layers, featuring 3 degrees of freedom, $W_{1}, W_{2}, W_{3}$, with $L_{x}=L_{y}=\lambda / 5$ and $h=\lambda / 20$ (Fig. 1); the macro-periodicity required to obtain the desired refraction is $\Lambda=1.2 \lambda$ (6 unit cells).

Our first goal is to compile the suitable LUT, associating all feasible triplets $\left(W_{1}, W_{2}, W_{3}\right)$ with their corresponding unitcell transmission and reflection coefficients. To use (1)-(3) to this end, we first need to establish a relation between the capacitor width $W$ and the effective load impedance-per-unitlength $\tilde{Z}$. This is done by simulating a single-layer structure in ANSYS HFSS for a range of $W$ values, and extracting via (2)-(3) with $N=1$ the complex $\tilde{Z}(W)$ leading to the recorded scattering parameters. Equipped with the interpolated relation $\tilde{Z}(W)$, we sweep the design space $\left(W_{1}, W_{2}, W_{3}\right)$ and use MATLAB to calculate for each triplet the predicted $R$ and $T$ following the scheme in Section III forming the desired LUT.

For refraction involving a normally incident plane wave, $0^{\circ}$ Huygens' meta-atoms can be used [3], corresponding to unit cells exhibiting unity transmission magnitude and linear phase across the period. Hence, we use the LUT to identify those combinations $\left(W_{1}, W_{2}, W_{3}\right)$ yielding the maximal transmission magnitude for every possible phase shift. The best unit cell geometries as a function of $\angle T$, obtained without any fullwave optimization, are presented in Fig. 2 (* markers), along with the predicted (orange circles) transmission magnitude and phase. The blue triangles denote the simulated values extracted for each of these meta-atoms from HFSS match these predictions well, indicating the fidelity of our analytical model, clearly capable of incorporating realistic copper losses.

Lastly, to form the desired MS, we sample Fig. 2 in $60 \mathrm{deg}$ intervals, choosing these 6 unit cells with the highest average $|T|$. This macro period, comprised of 30 copper capacitivelyloaded wires with the $W_{n}$ prescribed by the LUT, was simulated in HFSS under periodic boundary conditions. The recorded electric field distribution and the coupling efficiencies to the various FB modes are presented in Fig. 3 These results clearly indicate that although no full-wave optimization was performed, our semianalytical design scheme yielded a highly efficient refracting HMS. In fact, if one excludes the inevitable conductor losses, it is found that about $90 \%$ of the scattered power is coupled to the desired -1 mode in transmission, very close to the optimal coupling efficiency of $92 \%$ [3].

\section{CONCLUSION}

To conclude, we presented a rigorous semianalytical scheme for microscopic design of multilayered MSs. In contrast to

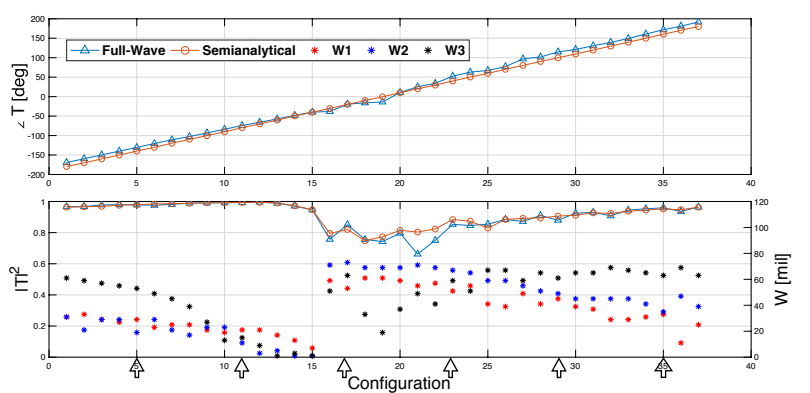

Fig. 2. Comparison between analytically predicted and full-wave simulated transmission (a) magnitude and (b) phase, for the capacitor widths $\left(W_{1}, W_{2}, W_{3}\right)$ denoted by * markers (Fig. 1 .

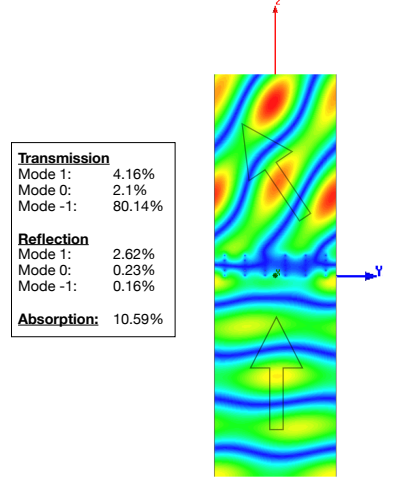

Fig. 3. Full-wave simulation results for the anomalous refraction HMS $\left(\theta_{\text {in }}=\right.$ $0^{\circ}, \theta_{\text {out }}=56^{\circ}$ ) semianlytically designed in Section III

previous techniques, the model properly accounts for interlayer near-field coupling, thus exhibiting high fidelity. Verified via commercial solvers, we expect this efficient methodology, avoiding full-wave optimization, to accelerate practical realizations of MSs for a variety of functionalities.

\section{REFERENCES}

[1] C. Pfeiffer and A. Grbic, "Metamaterial Huygens' surfaces: tailoring wave fronts with reflectionless sheets," Phys. Rev. Lett., vol. 110, no. 19, p. 197401, 2013.

[2] V. S. Asadchy, Y. Ra'di, J. Vehmas, and S. A. Tretyakov, "Functional metamirrors using bianisotropic elements," Phys. Rev. Lett., vol. 114, no. 9 , p. 095503, 2015.

[3] A. Epstein and G. V. Eleftheriades, "Huygens' metasurfaces via the equivalence principle: design and applications," J. Opt. Soc. Am. B, vol. 33, pp. A31-A50, Feb 2016.

[4] C. Pfeiffer and A. Grbic, "Bianisotropic metasurfaces for optimal polarization control: Analysis and synthesis," Phys. Rev. Appl., vol. 2, p. 044011 , Oct 2014.

[5] M. A. Cole, A. Lamprianidis, I. V. Shadrivov, and D. A. Powell, "Refraction efficiency of huygens' and bianisotropic terahertz metasurfaces," arXiv preprint arXiv:1812.04725, 2018.

[6] P. M. T. Ikonen, E. Saenz, R. Gonzalo, and S. A. Tretyakov, "Modeling and analysis of composite antenna superstrates consisting on grids of loaded wires," IEEE Trans. Antennas Propag., vol. 55, no. 10, pp. 2692 2700, 2007.

[7] A. Epstein and O. Rabinovich, "Perfect Anomalous Refraction with Metagratings," in 12th European Conference on Antennas and Propagation (EUCAP2018), arXiv: 1804.02362, 2018.

[8] Y. Ra'di, D. L. Sounas, and A. Alù, "Metagratings: Beyond the limits of graded metasurfaces for wave front control," Phys. Rev. Lett., vol. 119, no. 6, p. 067404, 2017. 